\title{
电离层二阶项延迟对 GPS 定位影响的分析模型与方法
}

\author{
刘西风 ${ }^{(12) *}$, 袁运斌 ${ }^{(1 *}$, 霍星亮 ${ }^{(1)}$, 李子申 ${ }^{(12)}$, 李薇 \\ (1) 中国科学院测量与地球物理研究所, 动力大地测量学重点实验室, 武汉 430077; \\ (2) 中国科学院研究生院, 北京 100049 \\ * 联系人, E-mail: liuxfshd@126.com; yybgps@ asch.whigg.ac.cn
}

2009-08-17 收稿, 2009-12-11 接受

国家杰出青年科学基金(批准号: 40625013)、国家自然科学基金(批准号: 40890160)、国家高技术研究发展计划(编号: 2007AA12Z311)和 中国科学院知识创新工程领域前沿项目(编号: 0909141014)资助

摘要 基于消除电离层一阶项延迟影响后的载波相位 $L c$ 线性组合观测量, 建立了电离层二 阶项延迟对 GPS 定位结果影响的定量分析模型. 在此基础上, 利用国际 GNSS 服务组织 WUHN 跟踪站连续 $15 \mathrm{~d}$ 的观测数据, 分析发现电离层二阶项延迟引起其定位结果规律性向 南偏移的现象; 结合武汉地区上空地磁场分布对电离层二阶项延迟的影响规律, 研究表明 地磁场是造成电离层二阶项延迟对该区域定位结果产生规律性向南偏移的主要原因之一。

\section{关键词}

全球定位系统

电离层二阶项延迟

总电子含量
随着以 GPS 为代表的多频多模全球导航卫星系 统(GNSS)的迅速发展与完善, 其应用水平与性能将 得到极大的提升. 然而, 电离层延迟仍是 GNSS 导航 与定位时的主要误差源之一, 特别是精密定位时必 须充分考虑其不利影响. 目前, 高精度 GNSS 用户大 多采用双频技术来削弱电离层延迟的影响, 但该技 术也仅能消除电离层一阶项延迟的影响. 随着多频 技术的出现，虽然理论上可以利用三频观测的线性 组合直接消除电离层一阶项延迟与二阶项延迟, 但 由于组合后的观测噪声影响放大了几十倍, 实际中 难以应用 ${ }^{[1 \sim 4]}$. 近年来, GNSS 技术越来越多应用于地 壳形变监测、地震灾害预测、板块运动等大地测量高 精度研究和应用领域时, 电离层高阶项延迟(主要是 二阶项)的影响备受关注与重视 ${ }^{[5 \sim 10]}$. 研究表明, 电 离层二阶项延迟对 GNSS 定位结果的影响可达厘米 级 ${ }^{[7 ~ 10]}$, 如何有效控制、改正甚至消除这一影响是 GNSS 技术实现优于厘米级绝对定位/㕰米级相对定位 等高精度测量服务必须解决的关键问题之一. 近年来, 国际上已有不少学者通过对高精度 GPS 观测站实测 数据的处理, 深人研究了电离层二阶项延迟对 GPS 定 位结果的影响, 大量数据处理结果表明电离层二阶项
延迟对一定区域范围内 GPS 定位结果的影响有一定 规律性 ${ }^{[7}$, 但尚未有人对这种规律性给予一种合理 的解释; 而在我国目前还没有相关研究报道.

本文以 GPS 为例, 基于消除电离层一阶项延迟 的双频载波相位 $L c$ 线性组合观测量, 建立了电离层 二阶项延迟对定位结果影响的定量分析模型. 基于 此模型, 利用国际 GNSS 服务组织 WUHN 跟踪站连 续 $15 \mathrm{~d}$ 的观测数据, 研究了武汉地区电离层二阶项 延迟对 GPS 定位结果的影响规律; 并结合国际地磁 场模型 IGRF10 分析了武汉地区上空的电离层二阶项 延迟空间几何分布规律; 初步研究表明地磁场分布 是武汉地区电离层二阶项延迟对 GPS 定位结果日均 变化影响呈偏南规律性偏移的主要原因之一.

\section{1 电离层二阶项延迟及其对 GPS 定位结果 的影响}

\subsection{GPS 观测中的电离层二阶项延迟}

(1)和(2)式分别给出了含有电离层二阶项延迟的 GPS 双频载波相位观测量 $L_{1}$ 与 $L_{2}$ 及其消除了电离层 一阶项的 $L c$ 线性组合 ${ }^{[5 \sim 7]}$ : 


$$
\begin{aligned}
L_{j} & =\rho-I_{L_{j}}^{(1)}-I_{L_{j}}^{(2)}+N_{L_{j}} \\
& =\rho-\frac{C_{x}}{2 f_{j}^{2}} \cdot S T E C-\frac{C_{x} \cdot C_{y}}{2 f_{j}^{3}} \cdot B \cdot \cos \theta \cdot S T E C+N_{L_{j}} \\
L_{c} & =\frac{f_{1}^{2} L_{1}-f_{2}^{2} L_{2}}{f_{1}^{2}-f_{2}^{2}}=\rho+I_{L c}^{(2)}+N_{c} \\
& =\rho+\frac{C_{x} \cdot C_{y}}{2 \cdot\left(f_{1}+f_{2}\right) \cdot f_{1} \cdot f_{2}} B \cos \theta \cdot S T E C+N_{c},
\end{aligned}
$$

其中, $f_{1}$ 与 $f_{2}$ 分别是 GPS 卫星信号的 2 个频率; $L_{j}(j=1,2)$ 分别是对应 GPS 信号两个频率上的相位 观测值 $(\mathrm{m}) ; L c$ 为 $L_{1}$ 和 $L_{2}$ 消电离层一阶项影响的线性 组合观测 $(\mathrm{m}) ; \rho$ 是测站与 GPS 卫星之间的站星距及 其他的非色散介质引起的误差项的组合 $(\mathrm{m}) ; N_{L_{j}}$ 表 示 $L_{j}$ 相位模糊度 $(\mathrm{m}) ; N_{c}$ 为 $N_{L_{j}}(j=1,2)$ 线性组合项, 且 $N_{c}=\left(f_{1}^{2} N_{L 1}-f_{2}^{2} N_{L 2}\right) /\left(f_{1}^{2}-f_{2}^{2}\right) ; I_{L j}^{(1)}, I_{L j}^{(2)}$ 分别表 示 $L_{j}$ 由电离层的一阶项、二阶项延迟引起的误差项 $(\mathrm{m})$; $I_{L c}^{(2)}$ 表示消电离层一阶项延迟后的 $L c$ 观测上残余的电 离层二阶项延迟 $(\mathrm{m}) ; C_{x}=80.6, C_{y}=2.801127 \times 10^{10}$, STEC 是 GPS 信号传播方向上的电离层电子总含量 $(\mathrm{TECU}) ; B$ 是站星连线与电离层薄层的交叉点处的地 磁感应强度 $(\mathrm{T}) ; \theta$ 是卫星信号矢量与地磁感应强度 矢量在电离层交叉点处的夹角 $\left(^{\circ}\right)$.

根据式(1)和(2), 将 GPS 载波相位 $L_{1}, L_{2}$ 及 $L c$ 线 性组合观测的电离层延迟简记为

$$
\begin{aligned}
& I_{L j}^{(1)}=\alpha_{j} \cdot S T E C, \quad(j=1,2), \\
& I_{L j}^{(2)}=\beta_{j} \cdot B \cos \theta \cdot S T E C, \\
& I_{L c}^{(2)}=2.0833 \times 10^{-16} \cdot B \cos \theta \cdot S T E C,
\end{aligned}
$$

其中, 常数 $\alpha_{1}=1.6237 \times 10^{-17}, \beta_{1}=2.8870 \times 10^{-16}$; $\alpha_{2}=2.6724 \times 10^{-17}, \beta_{2}=6.1029 \times 10^{-16}$.

在式(1) (5)中, $B$ 取值参考国际地磁场模型 IGRF10, 其量级一般在 $10^{-5} \mathrm{~T}$, 不超过 $10^{-4} \mathrm{~T}$; 地表 处 $B$ 值大小一般在 $2.5 \times 10^{-5} \sim 6.0 \times 10^{-5} \mathrm{~T}$, 其大小变化 为从赤道向两级逐渐增大 (http://www.ngdc.noaa.gov/ geomag/faqgeom.shtml); $\theta$ 值由 $B$ 矢量与信号矢量点 积计算而得; STEC 的计算在本文 2.2 节中进一步阐述. 由式(3)与(4), 可以得到 $L_{j}$ 观测量的电离层一阶项延 迟与二阶项延迟. 通过式(5), 可以估算消除电离层
一阶项延迟后的 $L c$ 观测中残余的电离层二阶项延迟, 在电离层较为活跃时其大小可达几个厘米 ${ }^{[7]}$.

\section{2 电离层二阶项延迟对 GPS 定位结果影响的定 量分析模型的建立}

本文主要研究消除电离层一阶项延迟影响的双 频载波相位组合观测 $L c$ 中的电离层二阶项延迟对 GPS 定位结果的影响. 考虑到电离层二阶项延迟 (模型)较其他误差源具有可分离性, 在不影响研究 结果的基础上, 忽略诸如卫星星历、卫星钟差、对 流层延迟、观测噪声以及相位模糊度等的影响, 所 以, 后面讨论与分析中将只顾及电离层二阶项延迟 误差.

电离层二阶项延迟对 GPS 定位结果影响如图 1 所示, 其中, $S(i)$ 表示可观测到的卫星 $(i=1,2, \cdots n, n$ 为同一历元所观测的卫星个数); $R$ 表示利用仅消除电 离层一阶项延迟的 $L c$ 观测得到的测站点位估值, 相 应卫星与 $R$ 之间的站星距为 $\gamma_{i} ; R^{\prime}$ 则表示对 $L c$ 观测中 残余的电离层二阶项延迟进行改正后得到的测站点 位估值，对应卫星与点位 $R^{\prime}$ 之间的站星距为 $\gamma_{i}^{\prime}$. 设 在以 $R$ 为原点的测站坐标系下卫星坐标为 $\left(X_{i}, Y_{i}, Z_{i}\right)$, $R^{\prime}$ 坐标为 $(x, y, z)$, 则对 $L c$ 观测中残余的电离层二阶 项延迟改正后引起的测站点位估值变化为 $\overrightarrow{R R^{\prime}}$, 其北 分量为 $x$, 东分量为 $y$, 垂直分量为 $z$; 分别定义受电离 层二阶项延迟影响与未受其影响的接收机钟差参数估 值 (与测站坐标同时解算)的大小为 $\delta T$ 与 $\delta T^{\prime} ; \overrightarrow{R R^{\prime}}$ 相 对于卫星两万多公里高度是个甚微量, 因此电离层二 阶项延迟对计算卫星相对测站的天顶距、方位角的影 响可以忽略. 令 $\theta_{i}, \alpha_{i}$ 分别为对应卫星的天顶距与方 位角, 则可得站星距 $\gamma_{i}, \gamma_{i}^{\prime}$ 的表达式:

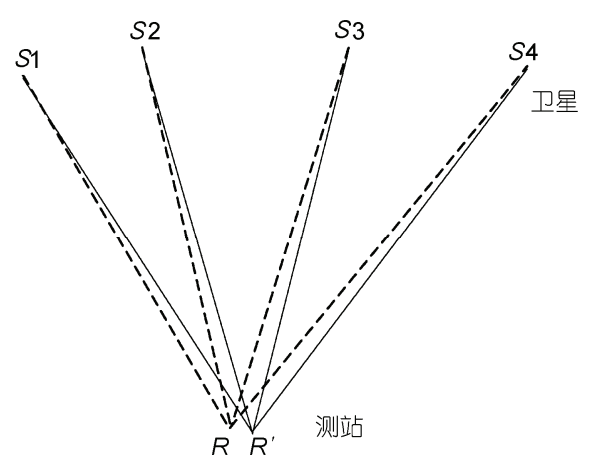

图 1 电离层二阶项延迟对 GPS 定位结果影响的示意图 


$$
\begin{aligned}
\gamma_{i}= & \sqrt{X_{i}^{2}+Y_{i}^{2}+Z_{i}^{2}}+\delta T \\
= & X_{i} \sin \left(\theta_{i}\right) \cos \left(\alpha_{i}\right)+Y_{i} \sin \left(\theta_{i}\right) \sin \left(\alpha_{i}\right) \\
& +Z_{i} \cos \left(\theta_{i}\right)+\delta T+\varepsilon_{i}, \\
\gamma_{i}^{\prime}= & \sqrt{\left(X_{i}-x\right)^{2}+\left(Y_{i}-y\right)^{2}+\left(Z_{i}-z\right)^{2}}+\delta T^{\prime} \\
= & \left(X_{i}-x\right) \sin \left(\theta_{i}\right) \cos \left(\alpha_{i}\right)+\left(Y_{i}-y\right) \sin \left(\theta_{i}\right) \sin \left(\alpha_{i}\right) \\
& +\left(Z_{i}-z\right) \cos \left(\theta_{i}\right)+\delta T^{\prime}+\varepsilon_{i}^{\prime},
\end{aligned}
$$

其中, $\varepsilon_{i}$ 和 $\varepsilon_{i}^{\prime}$ 为线性化误差(视为非常小的随机量, 通常远小于 $\left.I_{2}\right) ; \gamma_{i}$ 是 $L c$ 观测得到的站星距, $\gamma_{i}^{\prime}$ 是对 $\gamma_{i}$ 中残余的电离层二阶项延迟 $I_{L c, i}^{(2)}$ 进行改正后得到 的站星距. 实际计算中, 通常采用模型按照公式(5) 计算电离层二阶项延迟改正值 $\hat{I}_{L c, i}^{(2)}$, 它是理论值 $I_{L c, i}^{(2)}$ 的一个估值, 即 $\gamma_{i}=\gamma_{i}^{\prime}+I_{L c, i}^{(2)} \approx \gamma_{i}^{\prime}+\hat{I}_{L c, i}^{(2)}$.

式(6)与式(7)相减, 可得

$$
\begin{aligned}
\hat{I}_{L c, i}^{(2)}= & x \sin \left(\theta_{i}\right) \cos \left(\alpha_{i}\right)+y \sin \left(\theta_{i}\right) \sin \left(\alpha_{i}\right) \\
& +z \cos \left(\theta_{i}\right)+\delta t+\delta \varepsilon_{i}, \quad(i=1,2, \cdots, n),
\end{aligned}
$$

其中, $\delta \varepsilon_{i}=\varepsilon_{i}-\varepsilon_{i}^{\prime} ; \delta t=\delta T-\delta T^{\prime}$, 为改正电离层二 阶项延迟后引起接收机钟差估计值的变化.

针对同一历元观测到的每颗卫星都可给出类似与 式(8)的方程, 联合所有方程写成矩阵的形式如下:

$$
L=A X+\delta \varepsilon,
$$

其中,

$$
\begin{gathered}
L=\left(\hat{I}_{L c, 1}^{(2)}, \hat{I}_{L c, 2}^{(2)}, \cdots, \hat{I}_{L c, n}^{(2)}\right)^{\mathrm{T}}, \\
X=(x, y, z, \delta t)^{\mathrm{T}}, \\
\delta \varepsilon=\left(\delta \varepsilon_{1}, \delta \varepsilon_{2}, \cdots, \delta \varepsilon_{n}\right)^{\mathrm{T}},
\end{gathered}
$$

$$
A=\left(\begin{array}{cccc}
\sin \left(\theta_{1}\right) \cos \left(\alpha_{1}\right) & \sin \left(\theta_{1}\right) \sin \left(\alpha_{1}\right) & \cos \left(\theta_{1}\right) & 1 \\
\sin \left(\theta_{2}\right) \cos \left(\alpha_{2}\right) & \sin \left(\theta_{2}\right) \sin \left(\alpha_{2}\right) & \cos \left(\theta_{2}\right) & 1 \\
\ldots & \ldots & \ldots & \ldots \\
\sin \left(\theta_{n}\right) \sin \left(\alpha_{n}\right) & \sin \left(\theta_{n}\right) \sin \left(\alpha_{n}\right) & \cos \left(\theta_{n}\right) & 1
\end{array}\right) .
$$

基于最小二乘原理, 则可得到参数 $X$ 的估计式 如下:

$$
\hat{X}=\left(A^{\mathrm{T}} A\right)^{-1} A^{\mathrm{T}} L,
$$

利用式(10), 可计算出对残余的电离层二阶项延 迟影响改正后引起点位估值变化 $\overrightarrow{R R^{\prime}}$ 的北分量 $x$ 、东
分量 $y$ 、垂直分量 $z$ 以及接收机钟差变化 $\delta t$.

\section{2 实验与分析}

\section{1 实验概述}

基于国际 GNSS 服务组织 WUHN 跟踪站 2003 年 4 月 9 23 日连续 $15 \mathrm{~d}$ 的 GPS 双频实测数据, 通过 式(1)分析 $L_{1}$ 中电离层一阶项延迟以及二阶项延迟的 大小, 通过式(2)以消掉电离层一阶项延迟影响的 $L c$ 为例分析武汉地区电离层二阶项延迟对 GPS 定位结 果的影响. 其中, GPS 观测数据的采样间隔 $30 \mathrm{~s}$; 为 了避免多路径效应等与卫星观测高度角有关的误差 源影响, 卫星高度截止角设为 $20^{\circ}$.

\section{2 实验方法}

利用 GPS 载波相位与码观测量提取信号传播路 径上电离层 STEC, 相关方法详见文献[11 14]; 确定 $S T E C$ 后, 利用国际地磁场模型 IGRF10 计算电离层 交叉点处的地磁感强度 $\bar{B}$ 及其与卫星信号间的夹角 $\theta$, 基于式(3)和(4)即可计算出 GPS 载波相位 $L_{1}$ 观测 量中电离层一阶项与二阶项延迟大小, 结合式(5)和 式(10)可以逐历元计算 $L c$ 观测中的电离层二阶项延 迟对测站坐标与接收机钟差估值的影响.

\section{3 实验结果和分析}

按照 2.2 节给出的实验方法, 首先估计出 WUHN 测站观测卫星 $L_{1}$ 载波斜距方向上的电离层一 阶项延迟与二阶项延迟量, 进而深人讨论 $L c$ 观测量 中残余电离层二阶项延迟对武汉地区 GPS 定位结果 的影响.

(i ) 图 2 反映了 WUHN 测站观测到的相位 $L_{1}$ 上每一历元的观测卫星斜距方向上电离层一阶项延 迟与二阶项延迟连续 $15 \mathrm{~d}$ 时序变化. 在图 2 中, 横轴 均表示 2003 年 4 月 9 23 日间连续 $15 \mathrm{~d}$ 的时间变化; 图 2(a)与(b)的纵轴分别表示电离层一阶项延迟大小 与电离层二阶项延迟大小. 对比图 2(a)和(b)可以看 出, 电离层一阶项延迟与二阶项延迟均似有较显著 的周日变化特性, 但电离层二阶项延迟量级总体为 一阶项延迟量级的千分之一左右, 对信号传播的影 响最大可达几个厘米.

(ii) 图 3 反映了根据式(10)估算的电离层二阶 项延迟改正后引起的 WUHN 站坐标点位估值偏移在 

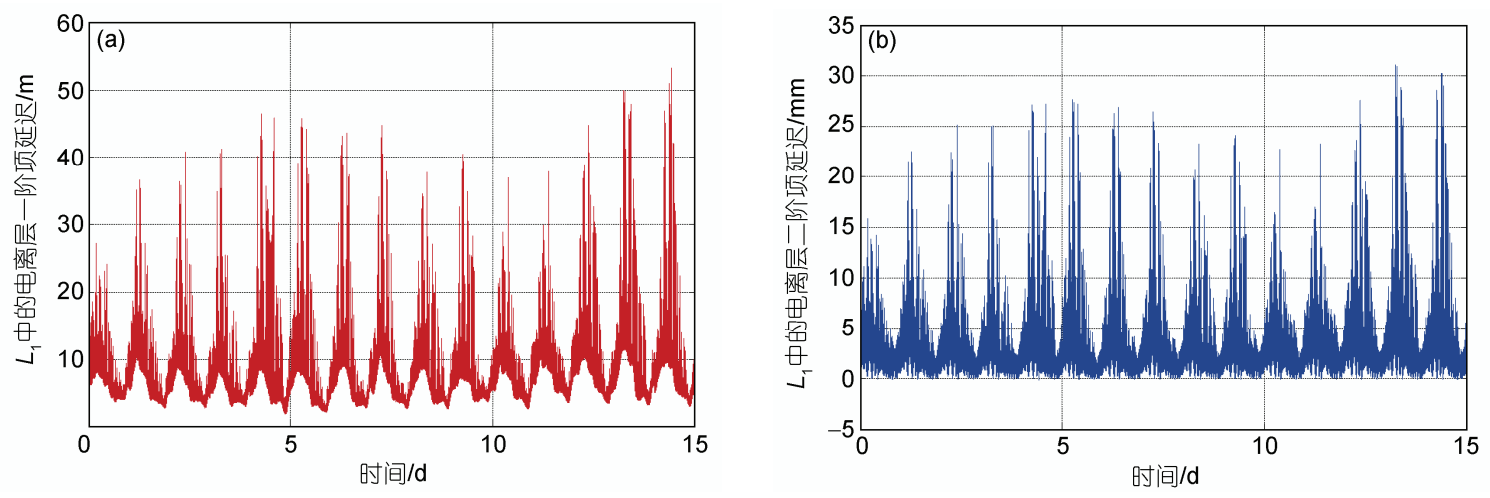

图 22003 年 4 月 9 23 日间 WUHN 站(观测到的所有卫星)相位信号 $L_{1}$ 上斜距方向的电离层不同阶项延迟时序变化图 (a) 一阶项延迟时序变化图; (b) 二阶项延迟时序变化图

北 $(\mathrm{d} N)$ 、东 $(\mathrm{d} E)$ 与垂直 $(\mathrm{d} H) 3$ 个方向及接收机钟差估 值变化 $(\mathrm{d} C)$ 的时序分布. 其中, 横轴表示上述连续 $15 \mathrm{~d}$ 的时间，纵轴表示点位估值变化矢量在测站坐标系 下各方向上的分量以及接收机钟差估值变化的大小. 从图 3 可以看出, 绿色虚线表示电离层二阶项延迟引 起的接收机钟差估值偏移大小的时间序列，量级达 厘米级, 数值基本为正, 即 $I_{L c}^{(2)}$ 改正后使得接收机钟 差估值偏大; 粉色实线表示由 $I_{L c}^{(2)}$ 引起的测站点位估 值在垂直方向上的时序变化, 量级达厘米级, 数值有 正有负, 但总体为负, 反映了点位估值垂向变化向下 偏移; 黑色点线 $\mathrm{d} N$ 表示对 $I_{L c}^{(2)}$ 改正后引起的测站点 位估值在水平面南方向分量上的时序变化，量级达 厘米级, 但其数值恒为负数, 即呈明显地向南偏移趋
势; 蓝色实线表示对 $I_{L c}^{(2)}$ 改正后引起的测站点位估值 在水平面东方向分量上的时序变化, 相对于其他分 量的变化而言, 量级很小, 日变化较为平稳, 表明电 离层二阶项延迟对测站点位估值变化在该方向上影 响较小, 且其数值正负不定. 可见, 对 $L c$ 观测量中残 余的电离层二阶项延迟改正后, WUHN 测站点位估 值变化在水平面上存在明显的向南偏移, 在垂直方 向上存在明显的降低, 在东西方向上变化则较小. 因 此, 在考虑优于厘米级绝对定位/毫米级相对定位高 精度要求时, 通常需要顾及并有效处理电离层二阶 项延迟的影响.

(iii) 表 1 进一步对图 3 探讨的 WUHN 站点位估 值偏差时间序列变化的日均变化进行统计. 其中, $A z$

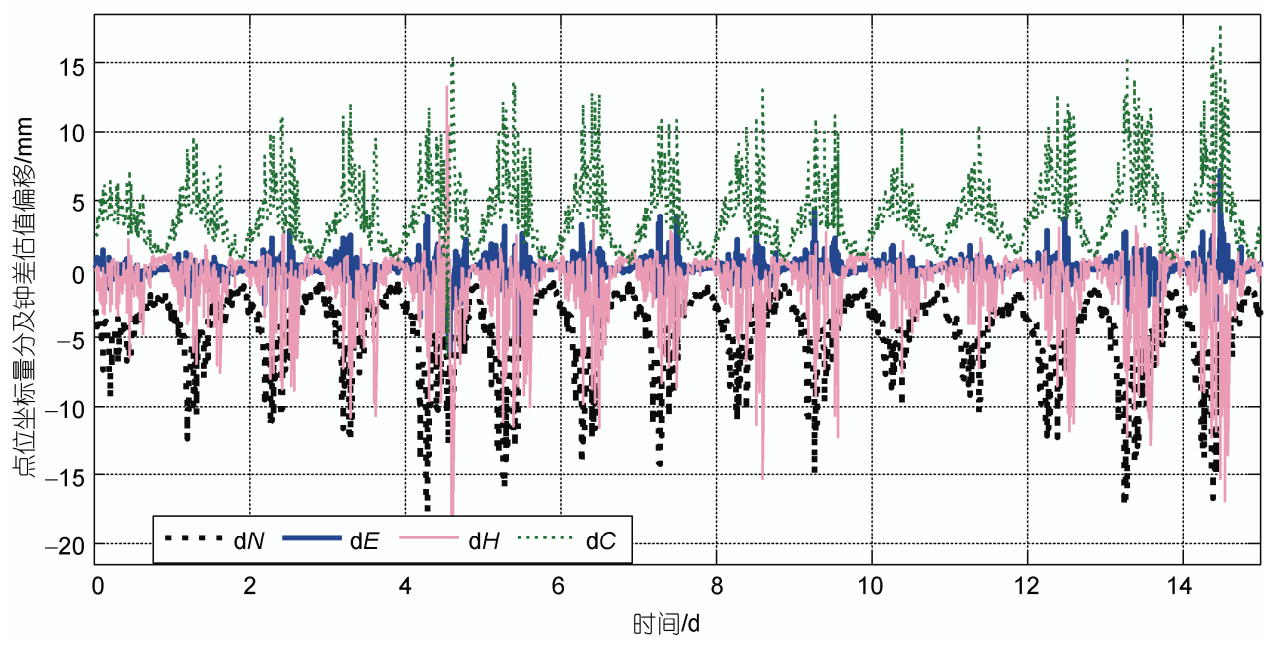

图 3 电离层二阶项延迟 $I_{L c}^{(2)}$ 引起的 WUHN 站点位坐标分量及接收机钟差估值偏移的时序分布图 $\mathrm{d} N$, 点位估值偏移北分量; $\mathrm{d} E$, 点位估值偏移东分量; $\mathrm{d} H$, 点位估值偏移垂直分量; $\mathrm{d} C$, 接收机钟差估值变化 
表示在以 $R$ 为原点的测站坐标系下, 根据式(10)计算 的点 $R^{\prime}$ 相对于测站 $R$ 的方位角; 天数 1 15: 表示上 述连续的 $15 \mathrm{~d}$.

表 1 中的 $\mathrm{d} E$ 量反映出 WUHN 测站由电离层二 阶项延迟引起的点位估值变化在东西方向上的偏移 量最大不超过 $0.5 \mathrm{~mm}$, 平均在东方向上偏移 0.12 $\mathrm{mm}$, 而 $\mathrm{d} N$ 量反映出的测站点位估值在南北方向上 的最大偏移量甚至达到近 $6 \mathrm{~mm}$ 多, 且均反映出坐标 向南偏移 ( $\mathrm{d} N$ 为负值). 也就是说, 电离层二阶项延迟 引起测站坐标在水平面上存在较大的向南偏移, 平 均向南偏移 $4.43 \mathrm{~mm} . \mathrm{d} C$ 量与 $\mathrm{d} H$ 量则反映出电离层 二阶项延迟也可导致接收机钟差估值及点位坐标在 垂向方向上的变化, 表中数据反映了武汉站二阶项 延迟的存在使得接收机钟差估值明显偏大, 点位估 值垂向变化呈较为明显向下的趋势. 特别需要指出 的是, $A z$ 量反映出电离层二阶项延迟引起的点位估 值变化在方位角上存在一定的规律, 即测站点位估 值偏南变化的方位角日间变化差异不大, 平均的方 位角为 $178.34^{\circ}$.

\section{3 武汉区域电离层二阶项延迟的空间分布 规律及对点位估值影响特征的估计}

由式(2)可以看出电离层二阶项延迟与地磁感应 强度 $B$ 密切相关. 地磁场对电离层二阶项延迟的影

\section{表 1 电离层二阶项延迟引起的武汉站定位结果日均变化} 矢量统计数据 ${ }^{\text {a) }}$

\begin{tabular}{cccccc}
\hline 时间/d & $\mathrm{d} N / \mathrm{mm}$ & $\mathrm{d} E / \mathrm{mm}$ & $\mathrm{d} H / \mathrm{mm}$ & $\mathrm{d} C / \mathrm{mm}$ & $A z /\left(^{\circ}\right)$ \\
\hline 1 & -3.66 & -0.04 & -0.83 & 3.43 & 180.59 \\
2 & -3.96 & -0.02 & -1.32 & 3.85 & 180.34 \\
3 & -3.84 & 0.22 & -1.33 & 3.71 & 176.64 \\
4 & -3.62 & 0.06 & -1.62 & 3.76 & 179.02 \\
5 & -5.42 & -0.22 & -3.09 & 5.37 & 182.31 \\
6 & -4.55 & 0.10 & -2.41 & 4.55 & 178.79 \\
7 & -4.31 & 0.18 & -1.61 & 4.08 & 177.61 \\
8 & -4.08 & 0.25 & -1.47 & 3.92 & 176.52 \\
9 & -3.92 & 0.13 & -1.54 & 3.86 & 178.05 \\
10 & -4.28 & 0.26 & -1.67 & 4.07 & 176.57 \\
11 & -3.77 & 0.19 & -1.05 & 3.64 & 177.10 \\
12 & -4.12 & 0.20 & -0.77 & 3.79 & 177.16 \\
13 & -5.03 & 0.25 & -1.73 & 4.77 & 177.17 \\
14 & -6.17 & -0.03 & -2.91 & 5.96 & 180.24 \\
15 & -5.68 & 0.29 & -2.60 & 5.43 & 177.04 \\
平均值 & -4.43 & 0.12 & -1.73 & 4.28 & 178.34 \\
\hline
\end{tabular}

a) $A z$ 表示在测站坐标系下测站点位估值变化的方位角, 如图 1 中 矢量 $\overrightarrow{R R^{\prime}}$ 相对于 $R$ 点的方位角
响具有什么规律? 在地磁场影响下, 电离层二阶项 延迟引起的点位坐标估值变化是否存在一定规律性? 为进一步研究地磁场对电离层二阶项延迟的影响特 性, 固定测站上空各交叉点处对应天顶方向上的 $\mathrm{TEC}$ 为 $100 \mathrm{TECU}^{[8]}$, 卫星相对于测站的天顶距以 $5^{\circ}$ 为间隔在 $0^{\circ} \sim 75^{\circ}$ 范围内取 16 组固定值, 分析电离层 二阶项延迟在假定仅受地磁场影响下的空间几何分 布规律. 图 4 给出了 2003 年 4 月 9 日仅在地磁场影 响下的武汉区域上空电离层二阶项延迟分布特征.

从图 4 中可以看到, 电离层二阶项延迟分布在东 西方向较为对称, 南北方向差异显著, 且南方位较北 方位出现的影响明显大得多; 电离层二阶项延迟正 负项出现情况及大小差异显著，负项出现仅局限在 偏北方位以及天顶距较大时. 武汉区域电离层二阶 项延迟分布规律反映在图形上的上述几何特征，可 以初步解释电离层二阶项延迟改正后引起的武汉地 区 GPS 定位结果平均变化偏南的现象(图 4 中绿色箭 头所示). 参考国际地磁场模型可知地磁场的日变化 乃至年变化甚微(http://www.ngdc.noaa.gov/geomag/ models.shtml), 即地磁感应强度 $B$ 的微量变化对分析 电离层二阶项延迟空间分布规律的影响甚小. 进一 步分析 2003 年 4 月 10 23 日武汉区域电离层二阶项 延迟空间分布规律与图 4 类似，限于篇幅，在此不一

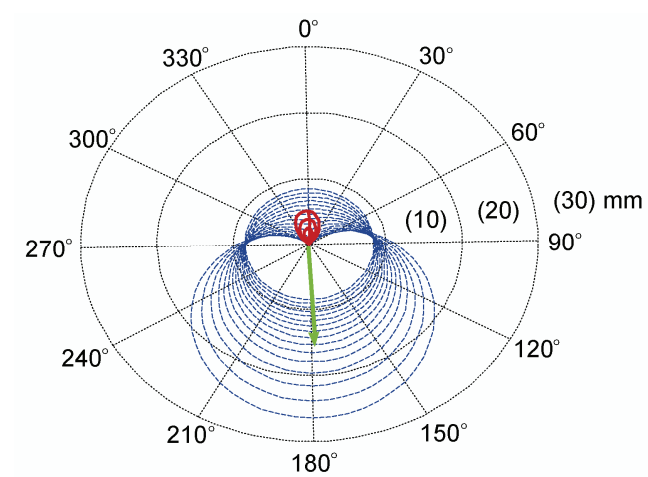

图 4 武汉站上空电离层二阶项延迟与卫星信号方位角的几 何关系及其对 GPS 定位结果影响特征的估计

测站交叉点处天顶方向上的 TEC 固定为 $100 \mathrm{TECU}$; 卫星相对测站 的天顶距值以 $5^{\circ}$ 为间隔在 $0^{\circ} \sim 75^{\circ}$ 范围内取 16 组固定值, 反映在图形 由内而外 $0^{\circ} \sim 75^{\circ}$ 依次对应 16 组值; 时间为 2003 年 4 月 9 日; $0^{\circ} \sim 360^{\circ}$ 表示卫星相对于测站的方位角; 图中括号内数据如(20)表示虚线圆 半径, 是电离层二阶项延迟绝对值的大小; 蓝折短线代表电离层二 阶项延迟为正，红实线则代表为负时的绝对值; 绿色箭头指向仅表 示 $L c$ 观测所得点位指向进行电离层二阶项延迟改正后所得 点位的矢量平面变化方向(如图 1 中矢量 $\overrightarrow{R R^{\prime}}$ 的平面指向) 
一列出. 以上分析表明地磁场是造成在电离层二阶 项延迟影响下定位结果平均变化呈一定规律性的主 要原因之一.

\section{4 结论}

基于消除电离层一阶项延迟影响后的载波相位 $L c$ 线性组合观测量, 建立了电离层二阶项延迟对 GPS 定位结果影响的定量分析模型与方法; 基于国 际 GNSS 服务组织 WUHN 跟踪站连续 $15 \mathrm{~d}$ 的双频实 测数据, 对比分析了电离层一阶项延迟与二阶项延 迟的量级大小, 重点研究了电离层二阶项延迟对
WUHN 测站 GPS 定位结果的影响特点. 研究结果表 明：电离层的二阶项延迟对武汉区域 GPS 点位估值 日均变化的影响反映在垂向上有较为明显的下降趋 势, 在水平面上向南移的趋势明显, 而东西方向上则 变化较小, 并且点位日均变化的方位相对稳定在正 南方向附近. 最后, 结合国际地磁场模型 IGRF10 分 析了仅在地磁场影响下的武汉地区电离层二阶项延 迟空间几何分布规律及对点位影响的变化特征, 并 推测地磁场是造成该区域电离层二阶项延迟引起 GPS 定位结果日均变化呈偏南规律性偏移的主要原 因之一.

致谢 IGS 国际 GNSS 组织提供了数据服务, 在完成过程中得到了张宝成博士和王海涛博士的热心帮助, 在此一并表示 感谢.

\section{参考文献}

1 李征航, 陈锴, 刘万科, 等. 顾及 $\mathrm{f}^{3}$ 项的电离层延迟模型. 武汉大学学报(信息科学版), 2007, 32: 139-143

2 刘琪, 张学军, 朱衍波. GPS 三频信息改正电离层折射误差高阶项的方法. 武汉大学学报(信息科学版), 2006, 9: 41-46

3 伍岳, 孟泱, 王泽民, 等. GPS 现代化后电离层折射误差高阶项的三频改正方法. 武汉大学学报(信息科学版), 2005, 30: 601一608

4 Wang Z M, Wu Y, Zhang K F, et al. Triple-frequency method for high-order ionospheric refractive error modeling in GPS modernization. J Glob Position Syst, 2005, 4: 291-295

5 Bassiri S, Hajj G A. Higher-order ionospheric effects on the global positioning system observables and means of modeling them. Manuscr Geod, 1993, 18: 280-289

6 Brunner F K, Gu M. An improved model for the dual frequency ionospheric correction of GPS observations. Manuscr Geod, 1991, 16: $205-214$

7 Kedar S, Hajj A, Wilson B D, et al. The effect of the second order GPS ionospheric correction on receiver positions. Geophys Res Lett, 2003, 30: 1829-1832

8 Hoque M M, Jakowski N. Mitigation of higher order ionospheric effects on GNSS users in Europe. GPS Solut, 2008, 12: 87—97

9 Munekane H. A semi-analytical estimation of the effect of second-order ionospheric correction on the GPS positioning. Geophys J Int, 2005, 163: 10-17

10 Morton Y T. Assessment of the Higher Order Ionosphere Error on Position Solutions. ION GNSS2008, Sep. 16-19, Savannah, Georgia, USA. 2008. 2361-2369

11 袁运斌, 霍星亮, 欧吉坤. 精确求定 GPS 信号的电离层延迟的模型与方法研究. 自然科学进展, 2006, 16: 40一48

12 Yuan Y B, Ou J K. An improvement to ionospheric delay correction for single frequency GPS user-the APR-I Scheme. J Geodesy, 2001, 75: 331-336

13 Yuan Y B, Ou J K. Auto-covariance estimation of variable samples (ACEVS) and its application for monitoring random ionosphere using GPS. J Geodesy, 2001, 75: 438—447

14 袁运斌. 基于 GPS 的电离层监测及延迟改正理论与方法的研究. 博士学位论文. 武汉: 中国科学院测量与地球物理研究所, 2002. $45-49$ 